\title{
Comparative evaluation of essential oils from Lippia javanica $L$ leaf obtained by two methods and their effect on Artemia salina L
}

\section{Oluwagbenga $O$ Adeogun, Alfred Maroyi*, Anthony Jide Afolayan}

Medicinal Plants and Economic Development Research Centre, Department of Botany, University of Fort Hare, Alice, Eastern Cape, South Africa

*For correspondence: Email: amaroyi@ufh.ac.za

\begin{abstract}
Purpose: To compare the chemical constituents of essential oils extracted from fresh and dried leaves of Lippia javanica by hydrodistillation (HD) and solvent-free microwave extraction methods (SFME), and evaluate their effects on Artemia salina.

Methods: Oil was extracted from the fresh and dried leaves of Lippia javanica by HD and SFME methods, and assayed for chemical constituents using gas chromatography-mass spectroscopy (GCMS). The oils were tested for hatchability and preliminary toxicity on Artemia salina for $72 h$. The lethal concentration required to kill fifty percent of $A$. salina $\left(L C_{50}\right)$ was determined by Probit regression analysis.

Results: Mesityl oxide was the most abundant compound in the essential oils. Mesityl oxide content of fresh and dried leaves extracted with HD was 25.33 and $29.83 \%$, respectively, while SFME method yielded 19.75 and $13.46 \%$, respectively. The average hatching success rate of the oil was $30 \%$ success while lethality was $100 \%$ after $72 \mathrm{~h}$. Median lethal concentration $\left(L C_{50}\right)$ of fresh and dried leaves extracted by HD was 90.11 and $128.49 \mu \mathrm{g} / \mathrm{mL}$, respectively, whereas SFME method resulted in $L C_{50}$ of 96.52 and $101.13 \mu \mathrm{g} / \mathrm{mL}$, respectively.

Conclusion: The results show that the essential oil yield is not significantly affected by the extraction methods used. However, the hatchability and lethality of the oils varied with the extraction method used
\end{abstract}

Keywords: Artemia salina, Lippia javanica, Essential oil, Hydrodistillation, Solvent-free microwave extraction

\begin{abstract}
This is an Open Access article that uses a funding model which does not charge readers or their institutions for access and distributed under the terms of the Creative Commons Attribution License (http://creativecommons.org/licenses/by/4.0) and the Budapest Open Access Initiative (http://www.budapestopenaccessinitiative.org/read), which permit unrestricted use, distribution, and reproduction in any medium, provided the original work is properly credited.
\end{abstract}

Tropical Journal of Pharmaceutical Research is indexed by Science Citation Index (SciSearch), Scopus, International Pharmaceutical Abstract, Chemical Abstracts, Embase, Index Copernicus, EBSCO, African Index Medicus, JournalSeek, Journal Citation Reports/Science Edition, Directory of Open Access Journals (DOAJ), African Journal Online, Bioline International, Open-J-Gate and Pharmacy Abstracts

\section{INTRODUCTION}

Lippia javanica (Burm f.) Spreng. (Verbenaceae), commonly known as lemon bush is indigenous to southern and tropical Africa [1]. Lippia javanica leaves and other aerial parts have a strong aromatic smell, the lemon-like fragrance is often given off when these plant parts are crushed [1]. Several authors have acknowledged its uses as an insect repellant, food preservative and in the treatment of a cough, fever, wounds, diarrhoea, chest pains and asthma [1,2-4]. Several authors 
$[1,3,5-7]$ have reported the presence of some essential oil constituents such as (Z)- $\beta$-ocimene, $\rho$-cymene, linalool, carvone, $\beta$-cubebene, tagetenone in the leaves of $L$. javanica. The presence of such constituents in the plant seems to ascribe aromatic fragrance to it $[1,8]$. Various works on essential oils have demonstrated that the presence of constituents in essential oils varies from one geographical zone to another and essential oil composition is also affected by time of harvest of the plant [3,7-9].

Essential oil predominantly consists of secondary metabolites that help the plants in self-defense against microbial attack [10]. The essential oils are conventionally distilled with hydrodistillation (HD), steam distillation or organic solvent extraction methods [11]. The use of these methods contributes to the degradation and loss of some volatile compounds in addition to the longtime of distillation $[10,11]$. There is need to address the inadequacy of hydrolytic processes of extraction of essential oils and this raised the need for an alternative to the use of SFME. Solvent-free microwave extraction processed the oils with the combination of microwave heating and distillation and this is done at atmospheric pressure. SFME has been used to distill essential oils from Origanum vulgare L., Cymbopogon citratus (DC.) Stapf, Mentha longifolia (L.) L., Moringa oleifera Lam. and several spices species $[12,13]$.

This study compared constituents of the essential oils generated through hydrodistillation and solvent-free microwave method of fresh and dried leaves and their toxicity on Artemia salina. Brine shrimp toxicity bioassay is a preliminary method of screening plant constituents for cytotoxicity and is an indicator for potential antitumor, anticancer and antimicrobial activities [11].

\section{EXPERIMENTAL}

\section{Plant material}

Fresh $L$. javanica leaves were harvested along the main access road to Hogsback. Hogsback is located at $32.5952^{\circ} \mathrm{S}, 26.9323^{\circ} \mathrm{E}$, close to Alice in the Eastern Cape Province, South Africa. Prof Maroyi of the Department of Botany, University of Fort Hare authenticated the plant and voucher specimen deposited in the Griffen Herbarium (UFH), University of Fort Hare. The leaves were separated from their branches and rinsed with distilled water, and then separated prior to extraction into fresh and dried samples. One of the dried samples was dried in an oven before analysis.

\section{Determination of dry leaf weight}

The dry weight of dried leaves was determined with $300 \mathrm{~g}$ of fresh leaves of $L$. javanica placed in an oven at $25{ }^{\circ} \mathrm{C}$ for $48 \mathrm{~h}$. The weight was obtained through evaporation of moisture from leaves of the plant. This was generated by subtracting the weight of the leaves after drying from the weight prior to oven drying of the leaves and then measured in percent.

\section{Solvent-free microwave extraction (SFME) of essential oil}

Solvent-free microwave extraction was carried out according to the method employed by Kayode and Afolayan [10]. Two hundred grams each of $L$. javanica fresh and dried leaves at different times were set into the reactor without the addition of water or any solvent. The exhaustive extraction of the essential oil was obtained at $40 \mathrm{~min}$.

\section{Hydrodistillation}

Two hundred grams each of fresh and dried $L$. javanica separately were hydrodistilled for $3 \mathrm{~h}$ in an all-glass Clevenger apparatus, with heat supplied to the heating mantle $\left(30{ }^{\circ} \mathrm{C}\right)$ and the essential oil was extracted with 4 litres of water for $3 \mathrm{~h}$ (until no more essential oil was recovered). The essential oil was collected and analysed immediately and this was done in accordance with the description of Okoh and Afolayan [11].

\section{Determination of yield of essential oil}

The yields of essential oils were determined using the method adopted by Adeogun et al [15] with slight modification. The quantity of the oil was obtained by deducting the weight of the dried essential oil over anhydrous sodium sulfate from the weight of the leaves prior to extraction and expressed as a percentage.

\section{Gas chromatography-mass spectroscopy (GC-MS)}

The GC-MS procedure used by Kayode and Afolayan [10] was adopted for this study. Agilent 6890 GC was coupled to an Agilent 5975 MSD with a Zebron-5MS column (ZB- 5MS $30 \mathrm{~m} \mathrm{x}$ $0.25 \mathrm{~mm} \times 0.25 \mathrm{Im})(5 \%$ phenylmethylpolysiloxane). GC grade helium was used as a carrier gas at a flow rate of $2 \mathrm{~mL} / \mathrm{min}$; splitless 1 $\mu \mathrm{L}$ injections were used. Injector temp $280^{\circ} \mathrm{C}$; source temp $280{ }^{\circ} \mathrm{C}$. Oven temp was $70{ }^{\circ} \mathrm{C}$, ramp $15^{\circ} \mathrm{C} / \mathrm{min}$ to $120^{\circ} \mathrm{C}$, ramp at $10^{\circ} \mathrm{C} / \mathrm{min}$ to $180^{\circ} \mathrm{C}$ then ramp at $20^{\circ} \mathrm{C} / \mathrm{min}$ to $270{ }^{\circ} \mathrm{C}$ and 
hold for 3 min. Data was gathered with Chemstation.

\section{Hatchability test on Artemia salina}

This test was conducted to ascertain if essential oils from Lippia javanica leaves has the potential of hatching the eggs of Artemia salina. Ten eggs were put into a $30-\mathrm{mL}$ capacity sterile petri dish, each containing a freshly prepared mixture of the essential oil solubilized with Dimethyl sulfoxide, and seawater $(\mathrm{pH}: 7.91)$ at varying concentration of $31.25,62.5,125,250,500,1000 \mu \mathrm{g} / \mathrm{ml}$. Different control samples consisting of $0.1 \%$ dimethyl sulphoxide (DMSO, i.e., $0.1 \mathrm{ml}$ DMSO in $100 \mathrm{ml}$ sea water), sea water and chloramphenicol were prepared on an individual base. The nauplii were counted in every 12 hours for 72 hours. The procedure for the experiment was carried out in a sterile petri-dish in triplicate, with access to illumination, and this followed the method adopted by Kayode and Afolayan [10] and Okoh and Afolayan [11].

\section{Lethality test on Artemia salina}

The test was performed to determine the effect of essential oil from fresh and dried leaves of $L$. javanica on brine shrimp nauplii. This was performed based on the method employed by Kayode and Afolayan [10] and Okoh and Afolayan [11] using brine shrimp eggs obtained from Ocean Star International, USA. The shrimp eggs were hatched in seawater for $48 \mathrm{~h}$ at $28^{\circ} \mathrm{C}$ with constant illumination prior to the addition of the test oil. The nauplii were attracted to one side of the vials with illumination. The stock solution of the essential oil from $L$. javanica was prepared by dissolving $100 \mathrm{mg}$ of the essential oil in 1.0 $\mathrm{mL}$ of DMSO. From the stock solution, $100 \mathrm{ml}$ of different concentrations of $31.25,62.5,125,250$, $500,1000 \mu \mathrm{g} / \mathrm{mL}$ of the essential oils was prepared with natural seawater. Control samples without essential oil consisting of $0.1 \%$ DMSO in sea water, sea water without essential oils and chloramphenicol were prepared differently. Ten nauplii were added to each test oil and each control sample and the dead nauplii were counted in every 12 hours for 72 hours. The test was conducted in triplicate with a sterile petridish, with access to illumination.

\section{Statistical analysis}

Statistical analysis was done using one-way analysis of variance (ANOVA) followed by Duncan multiple range test and probit regression analysis of the $\mathrm{LD}_{50}$ using SPSS. Significance of difference was set at $p<0.05$.

\section{RESULTS}

\section{Dry leaf weight}

The dry weight of the leaf after oven-drying was $49.37 \%$.

\section{Essential oil yield}

The yield of the essential oils from the leaf of $L$. javanica extracted with hydrodistillation and solvent-free microwave extractor is depicted in Table 1.

Table 1: Yield of essential oils of fresh and dried leaves of $L$. javanica

\begin{tabular}{lcc}
\hline Mode of extraction & $\begin{array}{c}\text { Fresh leaf } \\
(\%)\end{array}$ & $\begin{array}{c}\text { Dried leaf } \\
(\%)\end{array}$ \\
\hline Hydrodistillation & 1.52 & 0.42 \\
Solvent free microwave & 1.23 & 0.34 \\
extractor & & \\
\hline
\end{tabular}

\section{Chemical constituents of the essential oil}

The GC-MS analyses as shown in Table 2, revealed the presence of 44 and 56 compounds in oils extracted from fresh and dried leaves of $L$. javanica through hydrodistillation method respectively, while 48 and 44 compounds were extracted from fresh and dried leaves of $L$. javanica through solvent-free microwave extraction method. The Table also showed that 19 compounds occurred across the four test oils assayed.

Effect of the essential oils of different concentration on hatchability of Artemia salina at different time of exposure

Table 3 show the percentage hatchability of $A$. salina exposed to different time range. The percentage hatchability range from $10 \%$ after 12 hours exposure at a concentration of $500 \mu \mathrm{g} / \mathrm{mL}$ to $73.33 \%$ after $72 \mathrm{~h}$ at a concentration of 31 $\mu \mathrm{g} / \mathrm{mL}$. The figures show the significant difference $(p<0.05)$ based on DMRT on the hatchability activities of the different essential oils from fresh and dried leaves of $L$. javanica through hydrodistillation and solvent free microwave extraction methods with chloramphenicol treated samples, natural sea water and $0.1 \%$ DMSO.

Effect of lethality of the essential oils at different concentration on nauplii of Artemia salina at different time of exposure

The mortality of the nauplii of $A$. salina after exposure to different concentrations of the test oils as depicted in Table 4, which shows that the 
Table 2: Chemical constituents of the essential oil of fresh and dried leaves of Lippia javanica using hydrodistillation and solvent free microwave methods

\begin{tabular}{|c|c|c|c|c|c|}
\hline Essential oil constituent & $\begin{array}{l}\text { Fresh leaves } \\
\text { SFME (\%) }\end{array}$ & $\begin{array}{c}\text { Dried leaves } \\
\text { SFME (\%) }\end{array}$ & $\begin{array}{c}\text { Fresh leaves } \\
\text { HD (\%) }\end{array}$ & $\begin{array}{c}\text { Dried leaves } \\
\text { HD (\%) } \\
\end{array}$ & KI \\
\hline \multicolumn{6}{|l|}{$\begin{array}{l}\text { Monoterpene } \\
\text { hydrocarbons }\end{array}$} \\
\hline$\alpha$-Pinene & 0.08 & 0.12 & 0.30 & & 940 \\
\hline Sabinene & 2.50 & 0.19 & 4.25 & 0.33 & 955 \\
\hline$\beta$-Myrcene & 5.03 & 5.17 & 7.48 & 5.89 & 961 \\
\hline$\alpha$-Phellandrene & 3.12 & 2.59 & 5.03 & 3.71 & 968 \\
\hline$\rho$-Cymene & 1.49 & 1.31 & 2.22 & 1.09 & 976 \\
\hline$\alpha$-Ocimene & & 0.05 & 0.13 & 0.06 & 983 \\
\hline $\begin{array}{l}\text { 3-tert-butylphenol,m-tert- } \\
\text { butyl }\end{array}$ & 6.99 & 8.05 & 8.77 & & 1056 \\
\hline$\beta$ - Bourbonene & 0.28 & 0.21 & 0.55 & 0.24 & 1094 \\
\hline Y-Terpinene & 0.16 & 0.15 & 0.13 & 0.11 & 993 \\
\hline Caryophyllene & 2.22 & 1.18 & 2.37 & 3.12 & 1105 \\
\hline 4-tert-Butyphenol & 0.15 & & & 5.36 & 1080 \\
\hline Artemisia triene & 0.09 & & & & 1199 \\
\hline Geranyl-p-cymene & 0.09 & & & & 1175 \\
\hline (R)- $\alpha$-Pinene & & & 0.49 & & 940 \\
\hline Camphene & & & 0.11 & 0.05 & 947 \\
\hline$\beta$-Thujene & & 1.63 & 1.09 & 1.82 & 978 \\
\hline$\beta$ - Phellandrene & & & 3.19 & & 979 \\
\hline Cis- Verbenone & & & 0.15 & & 1052 \\
\hline 3,9- $\quad$ Epoxy-p-mentha-1,8 & & & 15.94 & 10.90 & 1062 \\
\hline \multirow{2}{*}{\multicolumn{6}{|c|}{$\begin{array}{l}\text { (10)-diene naphthalene, } \\
1,2,3,4, \quad 4 a, \quad 5, \quad 6, \quad 7- \\
\text { octahydro-4a-methyl }\end{array}$}} \\
\hline & & & & & \\
\hline Benzene, 1-ethoxy-4-ethyl- & & & 0.06 & & 1081 \\
\hline 2-Butanone, 4-phenyl & & & & 0.19 & 1037 \\
\hline Eugenol & & & & 0.19 & 1101 \\
\hline Isopiperitenone & & & 0.10 & 0.08 & 1098 \\
\hline \multicolumn{6}{|l|}{ Oxygenated monoterpenes } \\
\hline Mushroom alcohol & & 0.15 & & 0.10 & - \\
\hline Linalool & 4.76 & 3.92 & 4.71 & 2.00 & 1004 \\
\hline (+) Borneol & & 0.72 & & 0.09 & 1036 \\
\hline Terpene-4-ol & 0.37 & 0.41 & & & 1039 \\
\hline L-. $\alpha$-Terpineol & 1.32 & 0.85 & 1.13 & 0.50 & 1043 \\
\hline Isoborneol & 0.70 & & 0.54 & & 1038 \\
\hline (-) - Terpinene-4-ol & 0.69 & & & 0.60 & 1074 \\
\hline Geraniol & 0.08 & & & & 1499 \\
\hline Phytol & 0.21 & & & & 1232 \\
\hline 3,4-dimethylbenzyl alcohol & & & 0.19 & 0.11 & - \\
\hline Humulene & & & 0.17 & 0.19 & 1114 \\
\hline 6-epi-shyobunol & & & 0.07 & & 1132 \\
\hline 3 - Allylguaiacol & 0.63 & 0.34 & 0.18 & 0.05 & 1101 \\
\hline Isoborneol & 0.70 & & 0.54 & & 1038 \\
\hline \multicolumn{6}{|l|}{ Ketones } \\
\hline Artemia ketone & & 0.27 & & & 988 \\
\hline Thujone & 3.02 & 6.98 & & 0.23 & 1010 \\
\hline Mesityl oxide & 25.33 & 29.85 & 19.75 & 13.46 & - \\
\hline Isophorone & & & 1.19 & & 1103 \\
\hline \multicolumn{6}{|l|}{ Aldehydes } \\
\hline 2-Hexenal, (E) & 0.07 & & 0.20 & 0.15 & - \\
\hline \multicolumn{6}{|l|}{ Sequiterpene hydrocarbons } \\
\hline $\begin{array}{l}\text { Naphthalene, } 1,2,3,4,4 a, 5,6 \text {, } \\
\text { 7- octahydro-4a-methyl }\end{array}$ & 12.85 & 11.36 & & & 1060 \\
\hline Copaene & 0.49 & 0.44 & 0.61 & 0.53 & 1090 \\
\hline $\begin{array}{l}\text { 1,4,7, - Cycloundecatriene, } \\
\text { 1,5,9,9-tetramethyl-, Z,Z,Z- }\end{array}$ & 0.16 & 0.09 & & & 1114 \\
\hline$\beta$-Panasinsene & & 0.08 & & & 1116 \\
\hline
\end{tabular}


Table 2: Chemical constituents of the essential oil of fresh and dried leaves of Lippia javanica using hydrodistillation and solvent free microwave methods [continued...]

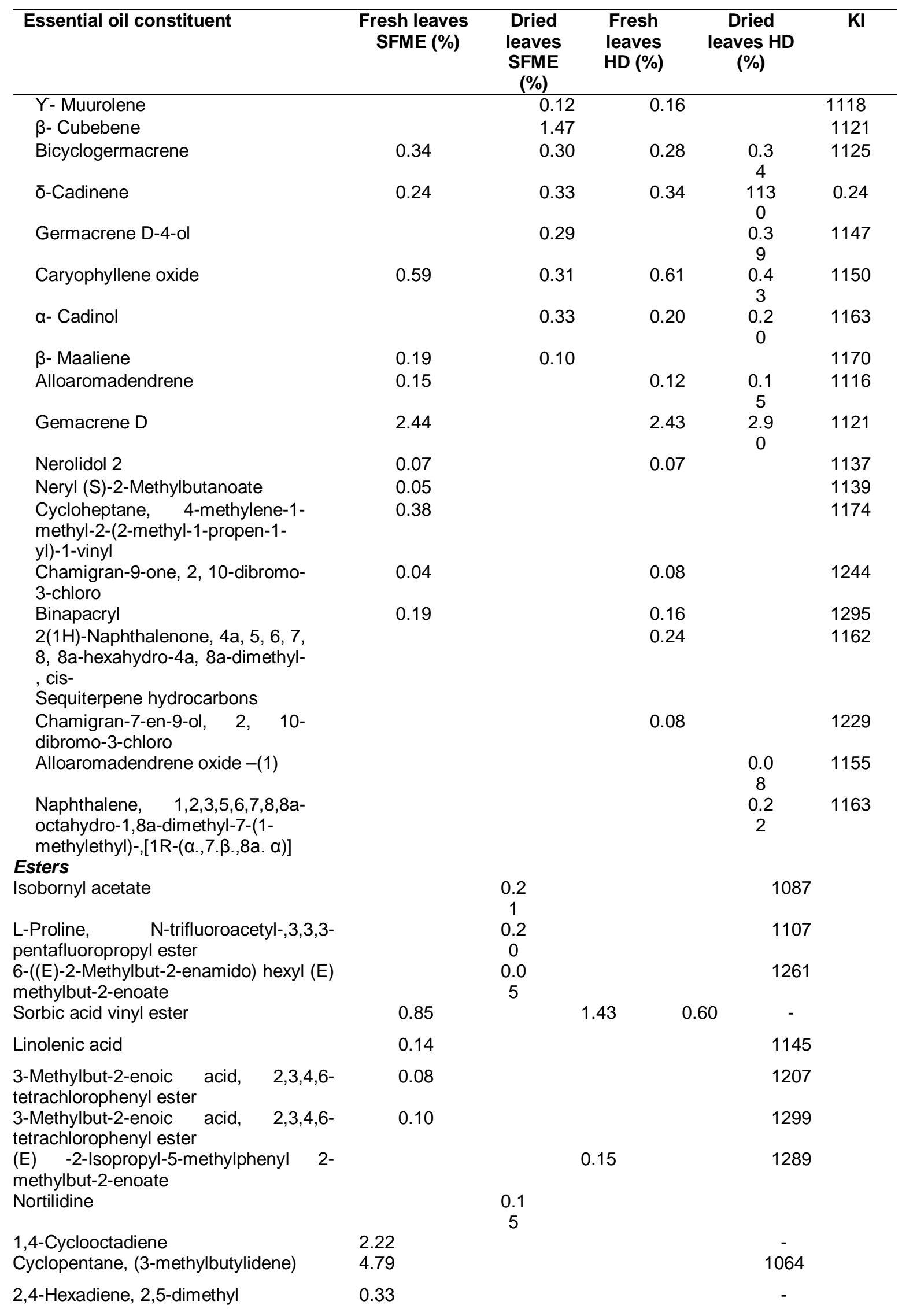


Table 2: Chemical constituents of the essential oil of fresh and dried leaves of Lippia javanica using hydrodistillation and solvent free microwave methods [continued...]

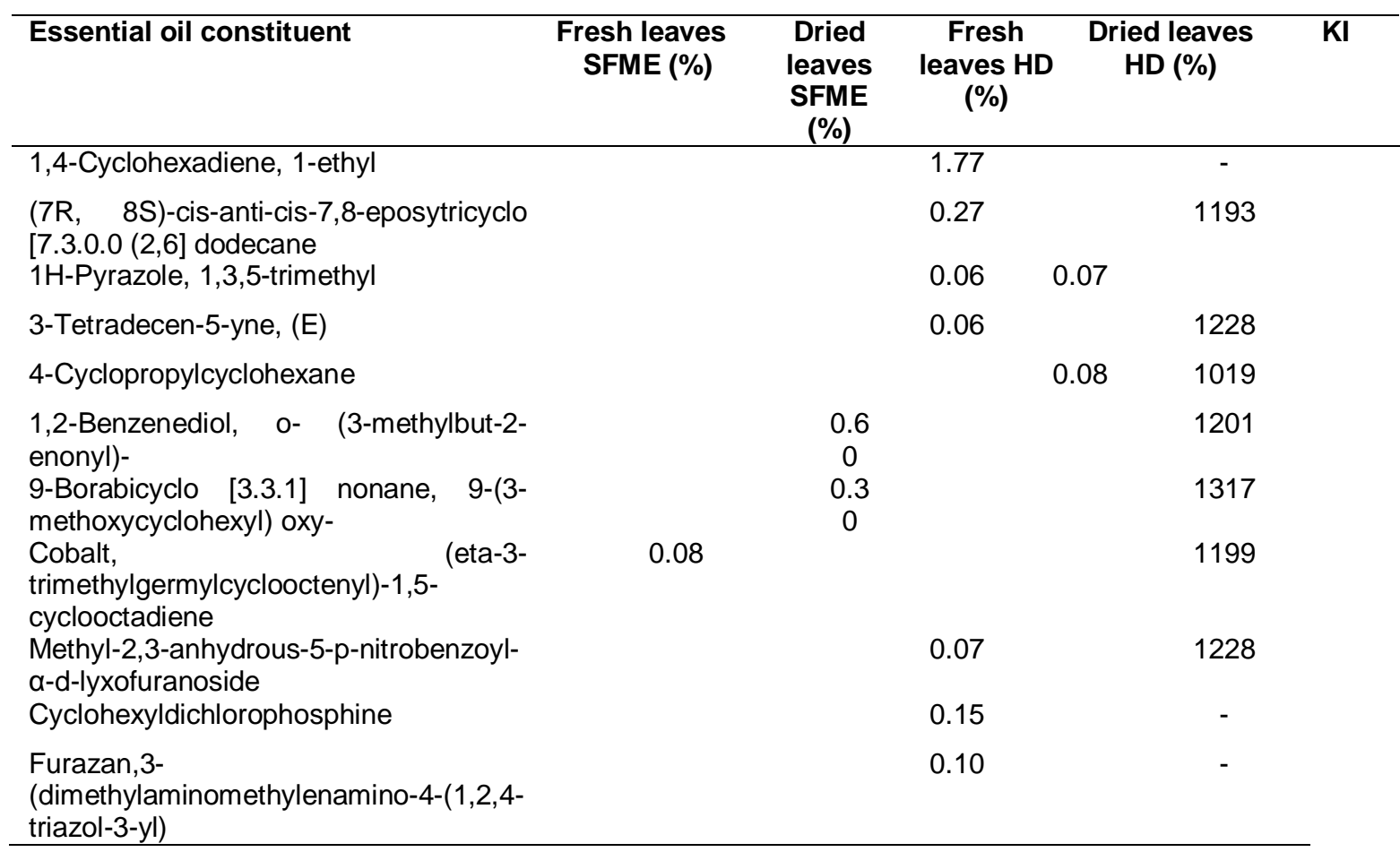

mortality ranged from $13.33 \%$ at $12 \mathrm{~h}$ exposure at a concentration of $31 \mu \mathrm{g} / \mathrm{mL}$ to $100 \%$ after 72 $\mathrm{h}$ exposure at a concentration of $1000 \mu \mathrm{g} / \mathrm{mL}$. There is a significant difference $(p<0.05)$ based on DMRT on the mortality activities of the test oils as against the mortality of $A$. salina in samples treated with chloramphenicol, natural sea water and $0.1 \%$ DMSO differently.

\section{Lethal concentration ( $\left(\mathrm{C}_{50}\right)$ of essential oils}

Table 5 shows the concentration required to kill half of the population of test $A$. salina. The $\mathrm{LC}_{50}$ of fresh and dried leaves extracted through hydrodistillation method is $90.11 \mu \mathrm{g} / \mathrm{mL}$ and $129.14 \mu \mathrm{g} / \mathrm{mL}$ respectively while the $L C_{50}$ of fresh and dried leaves extracted through solvent-free microwave extraction methods are $96.52 \mu \mathrm{g} / \mathrm{mL}$ and $101.13 \mu \mathrm{g} / \mathrm{mL}$ respectively. The study also takes cognizance of the $\mathrm{LC}_{50}$ of an antibiotic drug, chloramphenicol, which has an $\mathrm{LC}_{50}$ of $283.26 \mu \mathrm{g} / \mathrm{Ml}$. Fresh leaves SFME (\%) and dried leaves SFME (\%): composition of essential oils of fresh and dried leaves of $L$. javanica obtained using solvent free extraction method respectively. Fresh leaves HD (\%) and dried leaves HD (\%): The composition of essential oils of fresh and dried leaves of $L$. javanica obtained by hydrodistillation method, respectively. Compounds $<0.05 \%$ are not listed.

\section{DISCUSSION}

Several authors have documented the chemical constituents of essential oils from leaves of $L$. javanica $[1,3,6)$ but none has carried out a comparative evaluation of the composition of the essential oils from fresh and dried leaves of $L$. javanica using hydrodistillation and solvent free microwave extraction methods and their activities on $A$. salina. The dry weight of the leaf was 49.37\%; Alakali et al [16] observed that drying of plant samples above $50^{\circ} \mathrm{C}$ can affect the quality of the sample. The test plant leaves were dried at $30^{\circ} \mathrm{C}$ and this falls under what was established by Alkali et al [16].

This study was able to establish that the yield of the fresh leaves extracted with both SFME and $\mathrm{HD}$ had a higher yield than the yield of dried leaves through both SFME and HD. The higher yield in fresh leaves of the $L$. javanica negates what was reported by Silva et al [17], with the dried leaves of Eucalyptus cinera having a higher yield than the fresh ones. The yield of the essential oils of the leaf part of this plant was higher with hydrodistillation method compared with solvent-free microwave extraction method.

This observation corroborates the work of Kayode and Afolayan [10], they posited that the seed of Moringa oleifera had higher essential oils with HD than SFME. The work of Lucchesi et al [12] substantiates the high yield in oils extracted 
Table 3: Hatchability effect of essential oils from Lippia javanica on Artemia salina Nauplii

\begin{tabular}{|c|c|c|c|c|c|c|}
\hline \multirow[b]{2}{*}{$\begin{array}{l}\text { Test } \\
\text { sample }\end{array}$} & \multicolumn{6}{|c|}{ Hatchability success rate $(\%)$} \\
\hline & $31 \mu \mathrm{g} / \mathrm{mL}$ & $63 \mu \mathrm{g} / \mathrm{mL}$ & & & $500 \mu \mathrm{g} / \mathrm{mL}$ & $1000 \mu \mathrm{g} / \mathrm{mL}$ \\
\hline & \multicolumn{6}{|c|}{$12 \mathrm{~h}$} \\
\hline HDLD & $46.67 \pm 3.33^{\mathrm{c}}$ & $33.33 \pm 3.33^{b}$ & $26.67 \pm 3.33^{\mathrm{a}}$ & $23.33 \pm 3.33^{b}$ & $23.33 \pm 3.33^{\mathrm{c}}$ & $00.00 \pm 0.00^{\mathrm{a}}$ \\
\hline MCLD & $40.00 \pm 0.00^{b c}$ & $33.33 \pm 3.33^{b}$ & $26.67 \pm 3.33^{\mathrm{a}}$ & $20.00 \pm 0.00^{\mathrm{ab}}$ & $20.00 \pm 0.00^{b c}$ & $00.00 \pm 0.00^{a}$ \\
\hline MCLF & $30.00 \pm 5.77^{\mathrm{C}}$ & $26.67 \pm 3.33^{\mathrm{ab}}$ & $20.00 \pm 0.00^{\mathrm{a}}$ & $13.33 \pm 3.33^{\mathrm{a}}$ & $13.33 \pm 3.33^{\mathrm{ab}}$ & $00.00 \pm 0.00^{\mathrm{a}}$ \\
\hline HDLF & $36.67 \pm 3.33^{b c}$ & $30.00 \pm 0.00^{b}$ & $23.33 \pm 3.33^{a}$ & $16.67 \pm 3.33^{\mathrm{ab}}$ & $10.00 \pm 0.00^{\mathrm{a}}$ & $00.00 \pm 0.00^{a}$ \\
\hline DMSO & $20.00 \pm 3.33^{b c}$ & $20.00 \pm 0.00^{\mathrm{a}}$ & $20.00 \pm 0.00^{\mathrm{a}}$ & $20.00 \pm 0.00^{\mathrm{ab}}$ & $20.00 \pm 0.00^{\mathrm{bc}}$ & $20.00 \pm 0.00^{\mathrm{b}}$ \\
\hline sw & 43.67 & $43.67 \pm 3.33^{\mathrm{c}}$ & $43.67 \pm$ & $43.67 \pm$ & 43.67 & \\
\hline \multirow[t]{2}{*}{ CP } & $.33^{d}$ & $76.67 \pm 3.33^{d}$ & $76.67 \pm 3.33^{\mathrm{c}}$ & & $76.67 \pm 3.33^{\mathrm{e}}$ & $76.67 \pm 3.33^{d}$ \\
\hline & \multicolumn{6}{|c|}{$24 \mathrm{~h}$} \\
\hline HDLD & $60.00 \pm 0.00^{c}$ & $46.67 \pm 3.33^{\mathrm{abc}}$ & & & $16.67 \pm 3.33^{\mathrm{a}}$ & $00.00 \pm 0.00^{\mathrm{a}}$ \\
\hline MCLD & 53.3 & $50.00 \pm$ & & & & \\
\hline MCLF & $\pm 0.00^{\mathrm{a}}$ & 36.67 & & & & \\
\hline HDLF & 40.0 & $3.33^{\mathrm{b}}$ & $30.00 \pm$ & & & \\
\hline DMSO & 46.6 & $46.67 \pm$ & & & 46.6 & \\
\hline sw & & 53.3 & & & & \\
\hline \multirow[t]{2}{*}{$\mathrm{CP}$} & & $3.33^{d}$ & & & & \\
\hline & \multicolumn{6}{|c|}{$36 \mathrm{r}$} \\
\hline HDLD & & $00^{\mathrm{b}}$ & $3.33^{\mathrm{b}}$ & & & \\
\hline MCLD & $00^{c}$ & 56.6 & 50.0 & & & \\
\hline MCLF & $3^{\mathrm{a}}$ & 40.00 & 30.0 & & & \\
\hline HDLF & $3^{\mathrm{a}}$ & 36.67 & & & & \\
\hline DMSO & 50.00 & 50.00 & 50.0 & & & \\
\hline sw & $33^{\mathrm{bc}}$ & 56.6 & 56.6 & & & \\
\hline \multirow[t]{2}{*}{ CP } & & $73.33 \pm$ & $33^{d}$ & & & \\
\hline & \multicolumn{6}{|c|}{$48 \mathrm{~h}$} \\
\hline HDLD & & & & & $3^{\mathrm{ab}}$ & \\
\hline MCLD & & 60.00 & & & 20.0 & \\
\hline MCLF & & bcd & $0^{\mathrm{a}}$ & & 33.3 & \\
\hline HDLF & & ab & $3^{a}$ & & 33.3 & \\
\hline DMSO & & $3^{a}$ & & & 53.3 & \\
\hline sw & & $3^{c}$ & & & 66.6 & $.33^{\mathrm{e}}$ \\
\hline \multirow[t]{2}{*}{$\mathrm{CP}$} & $00 \pm 0.00^{b}$ & $.00 \pm 0.00^{d}$ & & $70.00 \pm 0.00^{c}$ & $70.00 \pm 0.00^{d}$ & $70.00 \pm 0.00^{\mathrm{e}}$ \\
\hline & \multicolumn{6}{|c|}{$60 \mathrm{~h}$} \\
\hline HDLD & & $66.67 \pm$ & $60.00 \pm 0.00^{\mathrm{ab}}$ & 53.33 & $46.67 \pm$ & \\
\hline MCLD & & $66.67 \pm$ & & & 30.0 & $3.33^{\mathrm{b}}$ \\
\hline MCLF & & $66.67 \pm 3.33^{\mathrm{ab}}$ & $53.33 \pm$ & 40.00 & 36.67 & 26.6 \\
\hline HDLF & & $42.00 \pm 2.80^{\mathrm{a}}$ & $56.67 \pm 3.33^{\mathrm{ab}}$ & $46.67 \pm 3.33^{\mathrm{ab}}$ & 30.00 & $3.33^{\mathrm{a}}$ \\
\hline DMSO & & $63.33 \pm 3.33^{\mathrm{ab}}$ & $63.33 \pm 3.33^{\mathrm{c}}$ & & $63.33 \pm 3.33^{\text {de }}$ & $63.33 \pm 3.33^{\mathrm{e}}$ \\
\hline sw & & & & & 70.00 & \\
\hline \multirow[t]{2}{*}{ CP } & $53.33 \pm 3.33^{\mathrm{a}}$ & $53.33 \pm 3.33^{\mathrm{ab}}$ & $53.33 \pm 3.33^{a}$ & $53.33 \pm 3.33^{b}$ & $53.33 \pm 3.33^{\mathrm{cd}}$ & $53.33 \pm 3.33^{d}$ \\
\hline & \multicolumn{6}{|c|}{$72 \mathrm{~h}$} \\
\hline HDLD & & & & & & \\
\hline & & & & & 40.00 & \\
\hline MCLF & & $73.33=$ & $66.67 \pm 3.33^{\mathrm{bc}}$ & & $50.00 \pm c$ & $40.00 \pm 0.00^{b}$ \\
\hline HDLF & & $70.00 \pm$ & 63.33 & $50.00 \pm$ & $36.67 \pm 6.67^{\mathrm{a}}$ & $\pm 3.33^{b}$ \\
\hline DMSO & & $73.33=$ & & 73.33 & & \\
\hline sw & & & & & & $76.67 \pm 3.33^{d}$ \\
\hline CP & $50.00 \pm 0.00^{\mathrm{a}}$ & $50.00 \pm 0.00^{\mathrm{a}}$ & $50.00 \pm 0.00^{\mathrm{a}}$ & $50.00 \pm 0.00^{\mathrm{ab}}$ & $50.00 \pm 0.00^{b}$ & $50.00 \pm 0.00^{\mathrm{c}}$ \\
\hline
\end{tabular}

with HD compared with less yield in SFME. They also mentioned that the high yield through HD was not quite significant considering that the time of heat takes up to $4.5 \mathrm{~h}$ and SFME only take 30 min to achieve the same purpose, which doesn't commensurate with the yield differences. 
Table 4: Lethality effect of essential oils from Lippia javanica on Artemia salina nauplii

\begin{tabular}{|c|c|c|c|c|c|c|}
\hline & \multicolumn{6}{|c|}{ Lethality effect (\%) } \\
\hline $\begin{array}{l}\text { Test } \\
\text { sample }\end{array}$ & $31 \mu g / m L$ & $63 \mu g / m L$ & $125 \mu \mathrm{g} / \mathrm{mL}$ & $250 \mu \mathrm{g} / \mathrm{mL}$ & $500 \mu \mathrm{g} / \mathrm{mL}$ & $1000 \mu \mathrm{g} / \mathrm{mL}$ \\
\hline & \multicolumn{6}{|c|}{$12 \mathrm{~h}$} \\
\hline HDLD & $13.33 \pm 3.33^{\mathrm{ab}}$ & $50.00 \pm 0.00^{c}$ & $60.00 \pm 0.00^{\mathrm{ca}}$ & $60.00 \pm 0.00^{c}$ & $66.67 \pm 3.33^{\mathrm{b}}$ & $80.00 \pm 0.00^{c}$ \\
\hline MCLD & $23.33 \pm 6.67^{\mathrm{ab}}$ & $46.67 \pm 3.33^{c}$ & $56.67 \pm 3.33^{\mathrm{ca}}$ & $70.00 \pm 0.00^{a}$ & $76.67 \pm 3.33^{\mathrm{D}}$ & $76.67 \pm 3.33^{c}$ \\
\hline MCLF & $26.67 \pm 3.33^{\mathrm{D}}$ & $46.67 \pm 3.33^{c}$ & $53.33 \pm 3.33^{c}$ & $70.00 \pm 0.00^{\mathrm{a}}$ & $76.67 \pm 3.33^{\mathrm{D}}$ & $83.33 \pm 3.33^{c}$ \\
\hline HDLF & $20.00 \pm 5.77^{\mathrm{ab}}$ & $50.00 \pm 0.00^{c}$ & $63.33 \pm 3.33^{a}$ & $73.33 \pm 3.33^{a}$ & $73.33 \pm 6.67^{\mathrm{D}}$ & $90.00 \pm 0.00^{a}$ \\
\hline DMSO & $10.00 \pm 0.00^{\mathrm{a}}$ & $10.00 \pm 0.00^{\mathrm{a}}$ & $10.00 \pm 0.00^{a}$ & $10.00 \pm 0.00^{\mathrm{a}}$ & $10.00 \pm 0.00^{\mathrm{a}}$ & $10.00 \pm 0.00^{a}$ \\
\hline SW & $13.33 \pm 3.33^{\mathrm{ab}}$ & $13.33 \pm 3.33^{\mathrm{a}}$ & $13.33 \pm 3.33^{\mathrm{ab}}$ & $13.33 \pm 3.33^{\mathrm{a}}$ & $13.33 \pm 3.33^{a}$ & $13.33 \pm 3.33^{a}$ \\
\hline \multirow[t]{2}{*}{ CP } & $20.00 \pm 0.00^{\mathrm{ab}}$ & $20.00 \pm 0.00^{\mathrm{D}}$ & $20.00 \pm 0.00^{\mathrm{D}}$ & $20.00 \pm 0.00^{\mathrm{b}}$ & $20.00 \pm 0.00^{a}$ & $20.00 \pm 0.00^{\mathrm{b}}$ \\
\hline & \multicolumn{6}{|c|}{$24 \mathrm{~h}$} \\
\hline HDLD & $33.33 \pm 3.33^{d}$ & $50.00 \pm 0.00^{a}$ & $50.00 \pm 0.00^{b}$ & $76.67 \pm 3.33^{\mathrm{d}}$ & $83.33 \pm 3.33^{b}$ & $86.67 \pm 3.33^{b}$ \\
\hline MCLD & $13.33 \pm 3.33^{a}$ & $50.00 \pm 0.00^{b}$ & $63.33 \pm 3.33^{\mathrm{bc}}$ & $66.67 \pm 3.33^{c}$ & $83.33 \pm 3.33^{b}$ & $86.67 \pm 3.33^{b}$ \\
\hline MCLF & $36.67 \pm 3.33^{d}$ & $63.33 \pm 3.33^{c}$ & $80.00 \pm 0.00^{\mathrm{d}}$ & $80.00 \pm 0.00^{d}$ & $86.67 \pm 3.33^{\mathrm{b}}$ & $86.67 \pm 3.33^{b}$ \\
\hline HDLF & $26.67 \pm 3.33^{\mathrm{bcd}}$ & $56.67 \pm 3.33^{\mathrm{bc}}$ & $66.67 \pm 3.33^{\mathrm{cd}}$ & $46.67 \pm 6.67^{\mathrm{b}}$ & $86.67 \pm 3.33^{b}$ & $90.00 \pm 0.00^{\mathrm{b}}$ \\
\hline DMSO & $26.67 \pm 3.33^{\mathrm{bcd}}$ & $26.67 \pm 3.33^{a}$ & $26.67 \pm 3.33^{\mathrm{a}}$ & $26.67 \pm 3.33^{a}$ & $26.67 \pm 3.33^{a}$ & $26.67 \pm 3.33^{a}$ \\
\hline SW & $20.00 \pm 0.00^{\mathrm{ab}}$ & $20.00 \pm 0.00^{\mathrm{a}}$ & $20.00 \pm 0.00^{a}$ & $20.00 \pm 0.00^{\mathrm{a}}$ & $20.00 \pm 0.00^{\mathrm{a}}$ & $20.00 \pm 0.00^{a}$ \\
\hline \multirow[t]{2}{*}{ CP } & $23.33 \pm 3.33^{\mathrm{bc}}$ & $23.33 \pm 3.33^{a}$ & $23.33 \pm 3.33^{a}$ & $23.33 \pm 3.33^{a}$ & $23.33 \pm 3.33^{a}$ & $23.33 \pm 3.33^{a}$ \\
\hline & \multicolumn{6}{|c|}{$36 \mathrm{~h}$} \\
\hline HDLD & $33.33 \pm 3.33^{\mathrm{D}}$ & $63.33 \pm 3.33^{c}$ & $76.67 \pm 3.33^{c}$ & $83.33 \pm 3.33^{c}$ & $90.00 \pm 0.00^{c}$ & $96.67 \pm 3.33^{c}$ \\
\hline MCLD & $46.67 \pm 3.33^{c}$ & $60.00 \pm 0.00^{c}$ & $76.67 \pm 3.33^{c}$ & $86.67 \pm 3.33^{c}$ & $90.00 \pm 0.00^{c}$ & $93.33 \pm 3.33^{c}$ \\
\hline MCLF & $16.67 \pm 3.33^{a}$ & $60.00 \pm 0.00^{c}$ & $70.00 \pm 0.00^{c}$ & $76.67 \pm 3.33^{c}$ & $86.67 \pm 3.33^{c}$ & $100.00 \pm 0.00^{c}$ \\
\hline HDLF & $43.33 \pm 2.24^{c}$ & $56.67 \pm 2.09^{c}$ & $70.00 \pm 0.00^{c}$ & $83.33 \pm 3.33^{c}$ & $86.67 \pm 3.33^{c}$ & $100.00 \pm 0.00^{C}$ \\
\hline DMSO & $33.33 \pm 3.33^{\mathrm{D}}$ & $33.33 \pm 3.33^{\mathrm{b}}$ & $33.33 \pm 3.33^{b}$ & $33.33 \pm 3.33^{\mathrm{D}}$ & $33.33 \pm 3.33^{\mathrm{D}}$ & $33.33 \pm 3.33^{\mathrm{D}}$ \\
\hline sw & $23.33 \pm 3.33^{\mathrm{ab}}$ & $23.33 \pm 3.33^{a}$ & $23.33 \pm 3.33^{a}$ & $23.33 \pm 3.33^{\mathrm{ab}}$ & $23.33 \pm 3.33^{\mathrm{a}}$ & $23.33 \pm 3.33^{a}$ \\
\hline CP & $30.00 \pm 0.00^{\mathrm{b}}$ & $30.00 \pm 0.00^{\mathrm{ab}}$ & $30.00 \pm 0.00^{\mathrm{ab}}$ & $30.00 \pm 0.00^{\mathrm{D}}$ & $30.00 \pm 0.00^{\mathrm{ab}}$ & $30.00 \pm 0.00^{\mathrm{a}}$ \\
\hline & & & & $48 \mathrm{~h}$ & & \\
\hline HDLD & $50.00 \pm 0.00^{\mathrm{a}}$ & $66.67 \pm 3.33^{c}$ & $76.67 \pm 3.33^{c}$ & $90.00 \pm 0.00^{\mathrm{a}}$ & $93.33 \pm 3.33^{c}$ & $100.00 \pm 0.00$ \\
\hline MCLD & $20.00 \pm 0.00^{\mathrm{a}}$ & $66.67 \pm 3.33^{c}$ & $73.33 \pm 3.33^{c}$ & $83.33 \pm 3.33^{c}$ & $90.00 \pm 0.00^{c}$ & $100.00 \pm 0.00$ \\
\hline MCLF & $36.67 \pm 3.33^{c}$ & $73.33 \pm 3.33^{c}$ & $80.00 \pm 0.00^{d}$ & $86.67 \pm 3.33^{\mathrm{cd}}$ & $96.67 \pm 3.33^{c}$ & $100.00 \pm 0.00$ \\
\hline HDLF & $53.33 \pm 3.33^{\mathrm{d}}$ & $73.33 \pm 3.33^{c}$ & $80.00 \pm 0.00^{d}$ & $90.00 \pm 0.00^{d}$ & $96.67 \pm 3.33^{c}$ & $100.00 \pm 0.00$ \\
\hline DMSO & $40.00 \pm 0.00^{c}$ & $40.00 \pm 0.00^{b}$ & $40.00 \pm 0.00^{\mathrm{b}}$ & $40.00 \pm 0.00^{\mathrm{b}}$ & $40.00 \pm 0.00^{\mathrm{b}}$ & $40.00 \pm 0.00$ \\
\hline SW & $30.00 \pm 0.00^{\mathrm{b}}$ & $30.00 \pm 0.00^{a}$ & $30.00 \pm 0.00^{\mathrm{a}}$ & $30.00 \pm 0.00^{\mathrm{a}}$ & $30.00 \pm 0.00^{\mathrm{a}}$ & $30.00 \pm 0.00$ \\
\hline \multirow[t]{2}{*}{ CP } & $30.00 \pm 0.00^{\mathrm{b}}$ & $30.00 \pm 0.00^{\mathrm{a}}$ & $30.00 \pm 0.00^{a}$ & $30.00 \pm 0.00^{a}$ & $30.00 \pm 0.00^{\mathrm{a}}$ & $30.00 \pm 0.00$ \\
\hline & \multicolumn{6}{|c|}{$60 \mathrm{~h}$} \\
\hline HDLD & $43.33 \pm 3.33^{c}$ & $80.00 \pm 0.00^{c}$ & $86.67 \pm 3.33^{\mathrm{c}}$ & $93.33 \pm 3.33^{\mathrm{cd}}$ & $100.00 \pm 0.00^{\mathrm{d}}$ & $100.00 \pm 0.00^{\mathrm{d}}$ \\
\hline MCLD & $20.00 \pm 0.00^{\mathrm{a}}$ & $73.33 \pm 0.33^{c}$ & $83.33 \pm 2.89^{c}$ & $86.67 \pm 2.24^{c}$ & $96.67 \pm 3.33^{d}$ & $100.00 \pm 0.00^{d}$ \\
\hline MCLF & $66.67 \pm 3.33^{a}$ & $76.67 \pm 3.33^{c}$ & $86.67 \pm 3.33^{c}$ & $96.67 \pm 3.33^{a}$ & $100.00 \pm 0.00^{\mathrm{a}}$ & $100.00 \pm 0.00^{\mathrm{a}}$ \\
\hline HDLF & $70.00 \pm 0.00^{\mathrm{a}}$ & $80.00 \pm 0.00^{c}$ & $90.00 \pm 0.00^{c}$ & $100.00 \pm 0.00^{a}$ & $100.00 \pm 0.00^{\mathrm{a}}$ & $100.00 \pm 0.00^{\alpha}$ \\
\hline DMSO & $43.33 \pm 3.33^{c}$ & $43.33 \pm 3.33^{\mathrm{D}}$ & $43.33 \pm 3.33^{\mathrm{D}}$ & $43.33 \pm 3.33^{\mathrm{D}}$ & $43.33 \pm 3.33^{c}$ & $43.33 \pm 3.33^{c}$ \\
\hline sw & $30.00 \pm 0.00^{\mathrm{b}}$ & $30.00 \pm 0.00^{\mathrm{a}}$ & $30.00 \pm 0.00^{a}$ & $30.00 \pm 0.00^{\mathrm{a}}$ & $30.00 \pm 0.00^{a}$ & $30.00 \pm 0.00^{a}$ \\
\hline \multirow[t]{2}{*}{ CP } & $36.67 \pm 3.33^{\mathrm{bc}}$ & $36.67 \pm 3.33^{a}$ & $36.67 \pm 3.33^{\mathrm{ab}}$ & $36.67 \pm 3.33^{\mathrm{ab}}$ & $36.67 \pm 3.33^{\mathrm{D}}$ & $36.67 \pm 3.33^{\mathrm{D}}$ \\
\hline & \multicolumn{6}{|c|}{$72 \mathrm{~h}$} \\
\hline HDLD & $70.00 \pm 0.00^{\mathrm{b}}$ & $80.00 \pm 0.00^{\mathrm{b}}$ & $90.00 \pm 0.00^{\mathrm{D}}$ & $100.00 \pm 0.00^{c}$ & $100.00 \pm 0.00^{\mathrm{D}}$ & $100.00 \pm 0.00^{\mathrm{b}}$ \\
\hline MCLD & $73.33 \pm 3.33^{\mathrm{D}}$ & $83.33 \pm 3.33^{\mathrm{D}}$ & $90.00 \pm 0.00^{\mathrm{b}}$ & $100.00 \pm 0.00^{c}$ & $100.00 \pm 0.00^{\mathrm{b}}$ & $100.00 \pm 0.00^{\mathrm{b}}$ \\
\hline MCLF & $36.67 \pm 3.33^{a}$ & $80.00 \pm 0.00^{\mathrm{b}}$ & $90.00 \pm 0.00^{\mathrm{b}}$ & $90.00 \pm 0.00^{\mathrm{b}}$ & $100.00 \pm 0.00^{\mathrm{b}}$ & $100.00 \pm 0.00^{\mathrm{b}}$ \\
\hline HDLF & $46.67 \pm 3.33^{a}$ & $86.67 \pm 3.33^{b}$ & $90.00 \pm 0.00^{b}$ & $93.33 \pm 3.33^{\mathrm{bc}}$ & $100.00 \pm 0.00^{b}$ & $100.00 \pm 0.00^{b}$ \\
\hline DMSO & $40.00 \pm 5.77^{\mathrm{a}}$ & $40.00 \pm 5.77^{a}$ & $40.00 \pm 5.77^{a}$ & $40.00 \pm 5.77^{a}$ & $40.00 \pm 5.77^{a}$ & $40.00 \pm 5.77^{a}$ \\
\hline SW & $36.67 \pm 3.33^{a}$ & $36.67 \pm 3.33^{a}$ & $36.67 \pm 3.33^{a}$ & $36.67 \pm 3.33^{a}$ & $36.67 \pm 3.33^{a}$ & $36.67 \pm 3.33^{a}$ \\
\hline CP & $40.00 \pm 0.00^{\mathrm{a}}$ & $40.00 \pm 0.00^{\mathrm{a}}$ & $40.00 \pm 0.00^{\mathrm{a}}$ & $40.00 \pm 0.00^{\mathrm{a}}$ & $40.00 \pm 0.00^{\mathrm{a}}$ & $40.00 \pm 0.00^{\mathrm{a}}$ \\
\hline
\end{tabular}

This study reported the identification of 104 compounds from the fresh and dried leaves of $L$. javanica using HD and SFME. Maroyi [1] reported on his work the presence of 133 compounds in the oils of $L$. javanica regardless of the method of extraction. The number of compounds identified in the essential oils of test L. javanica is 44, 56, 48 and 44 in fresh and dried leaves extracted through HD and fresh and dried leaves through SFME respectively. The presence of more compounds in the dried leaves of $L$. javanica extracted through hydro distillation in 
Table 5: Lethality index (indicated by $\mathrm{LC}_{50}$ ) of test oils extracted from L. javanica on Artemia salina

\begin{tabular}{lccccc}
\hline Treatment & $\mathbf{L C}_{\mathbf{5 0}}(\boldsymbol{\mu g} / \mathbf{m l})$ & Regression equation & $\mathbf{R}^{\mathbf{2}}(\%)$ & $\boldsymbol{P}$-value & Chi-square \\
\hline HDLD & 129.14 & $\mathrm{Y}=1.25 \mathrm{X}+-2.75$ & 80 & 0.0 & 22.19 \\
HDLF & 90.11 & $\mathrm{Y}=1.25 \mathrm{X}+-2.25$ & 91.2 & 0.1 & 12.58 \\
MCLD & 101.13 & $\mathrm{Y}=1 \mathrm{X}+-2$ & 90.6 & 0.8 & 8.33 \\
MCLF & 96.52 & $\mathrm{Y}=1 \mathrm{X}+-2$ & 97.6 & 0.7 & 2.36 \\
CP & 283.26 & $\mathrm{Y}=0.0032 \mathrm{X}+0.9$ & 77.2 & 0.15 & 3.76 \\
\hline
\end{tabular}

$R^{2}$ : coefficient of determination of regression equation; $p$-values indicate the level of significance of the regression equation; values less than 0.05 are significant and those less than 0.05 are not significant at $5 \%$ level of probability

this study support the assertion raised by Asekun et al [18] that dried plants yielded more oils than the fresh plants. It was noted in the study that 19 compounds were present in all test oils. The essential oils were dominated by sesquiterpene hydrocarbon, considerable amounts of monoterpene and oxygenated hydrocarbons, and ketones.

The presence of mesityl oxide, $\rho$-terpinene, $\rho$ cymene, thujone and some other chemical constituents in all test oils showed that hydrolysis is not outrightly a significant factor that influences the processing of compounds in essential oils $[10,19]$ and high compositions of some of these compounds have been attributed to ethnopharmacological, food preservation and flavouring activities. The low yield in test oils extracted from the dried leaves of $L$. javanica compared to the fresh leaves of $L$. javanica can be ascribed to the drying of the leaves before extraction. This assertion conformed to the earlier work by Rahimmalek et al [20] that attributed the reduced yield of essential oils from the leaves of Thymys daenensis was to drying of the leaves before distillation.

The presence of naphthalene, 1, 2, 3, 4, 4a, 5, 6, 7- octahydro-4a-methyl and some other compounds only in the essential oils from fresh and dried leaves of $L$. javanica through SFME method might be ascribable to the reduction in thermal and hydrolytic effect compared with hydrodistillation that that uses a great amount of water, time and energy [12].

The hatchability results obtained showed that the test oils had paltry inhibition on the $A$. salina. The hatchability successes in test oils extracted from the dried leaves of $L$. javanica through HD and SFME at $31 \mu \mathrm{g} / \mathrm{mL}$ were $73.33 \%$, followed by 70 and $52.33 \%$ in fresh leaves through SFME and $\mathrm{HD}$ respectively. The significant low hatchability activity of essential oil of the fresh leaves of $L$. javanica extracted through HD might be due to the presence and yield of some inhibitory compounds such as humulene, 6-epi-shybunol, 3-Tetradecen-5-yne, Cyclopropanecarboxylic acid. Silva et al [17] and
Bartololme et al [21] established the contributory effects of 3- tetradecen-5-yne and humulene as part of the compounds responsible for the anticancer and antitumoral activities of Bidens pilosa L. and Casearia sylvestris Sw.

The number of eggs hatched decreased with increasing concentration of the essential oils of $L$. javanica at a different time of exposure, mostly because of the ability of the varying concentration of test oil to diffuse across the shell of the eggs and inhibit the development of $A$. salina fetus [22]. The hatched egg increased with increase in time of exposure and the very low hatching success recorded at 12 or $24 \mathrm{~h}$ of exposure can be attributed to the alteration of the development of $A$. salina embryos because of the vulnerability of the organism to toxins at earlier developmental stages [23]. The rate of activities of the test oil on mortalities of $A$. salina, depend on the status and method of extraction from the leaf of $L$. javanica.

The evaluated mortality of $A$. salina at a different time of exposure showed that the fresh leaf extracted through HD had a high mortality rate, followed by fresh leaf through SFME, dried leaf through HD and dried leaf through SFME. Mortality increased with an increase in the concentration of different test oils, with mortality of fresh leaf obtained by $\mathrm{HD}$ at $31 \mu \mathrm{g} / \mathrm{mL}$ being $26.67 \%$ while at $1000 \mu \mathrm{g} / \mathrm{mL}$, it was $90.0 \%$, after $24 \mathrm{~h}$ exposure.

Kayode and Afolayan [10], and Okoh and Afolayan [11] posited that increase in concentration affected the mortality rates of essential oils from the leaves of Mentha longiflora and the seeds of Moringa oleifera on $A$. salina. They concluded that with a lower concentration, there is less mortality of $A$. salina.

$\mathrm{LC}_{50}$ values (fresh leaf $(\mathrm{HD}): 90.11 \mu \mathrm{g} / \mathrm{mL}$, dried leaf (HD): $129.11 \mu \mathrm{g} / \mathrm{mL}$, fresh leaf (SFME): $96.52 \mu \mathrm{g} / \mathrm{mL}$ and dried leaf (SFME): 101.13 $\mu \mathrm{g} / \mathrm{mL}$ ) obtained after $24 \mathrm{~h}$ exposure showed that all test oils were moderately toxic on $A$. salina. The moderate toxicity status of the test oils was deduced from Clarkson's toxicity index. 
Clarkson's toxicity index placed $\mathrm{LC}_{50}<1000$ $\mu \mathrm{g} / \mathrm{mL}$ as being toxic, $\mathrm{LC}_{50}$ of $500-1000 \mu \mathrm{g} / \mathrm{mL}$ as low toxic, $\mathrm{LC}_{50}$ of $100-500 \mu \mathrm{g} / \mathrm{mL}$ as medium toxic while $\mathrm{LC}_{50}$ of $100-500 \mu \mathrm{g} / \mathrm{mL}$ as being non- toxic. The $\mathrm{LC}_{50}$ was determined after $24 \mathrm{~h}$ based on precedence laid by Hamidi et al [24] and Adeogun et al [15] that most toxicity studies which use the Brine shrimp assay determined the toxicity by counting the survived nauplii after 24 hours of exposure to the tested sample.

The high mortality rate of the test oils corroborates the earlier work by Okoh and Afolayan [10] that recorded $\mathrm{LC}_{50}$ of 54.4 and 77.5 $\mu \mathrm{g} / \mathrm{ml}$ for SFME and HD of essential oils from Mentha longifolia L. leaf. They concluded secondary metabolites from plants, which are active medicinally, are most times more toxic to brine shrimps. Hamidi et al [24] also made mention that the toxicity of plants may originate from different contaminants or from plant chemical compounds that are part of the plant.

\section{CONCLUSION}

The findings of this study show that the essential oil yield is not dependent on the method of extraction. However, solvent-free microwave method of extraction saves time and energy. The toxicity of the oil towards Artemia salina is moderate. The essential oils at low concentrations can be developed for enhancement of shelf life of food and for the treatment of flu and malaria.

\section{DECLARATIONS}

\section{Acknowledgement}

The authors greatly appreciate Govan Mbeki Research and Development Centre (GMRDC) of the University of Fort Hare, South Africa, for funding this project and the University of Lagos, Nigeria for granting Dr OO Adeogun study leave to utilize the funds to carry out the project at the University of Fort Hare.

\section{Conflict of Interest}

No conflict of interest associated with this work.

\section{Contribution of Authors}

The authors declare that this work was done by the authors named in this article and all liabilities pertaining to claims relating to the content of this article will be borne by them.

\section{REFERENCES}

1. Maroyi A. Lippia javanica (Burm .f.) Spreng: Traditional and Commercial Uses and Phytochemical and Pharmacological Significance in the African and Indian Subcontinent. Evid Based Comple Alternat Med 2017; ID 6746071: 1-34.

2. Linde JH, Combrinck $S$, Reigner TJC, Virijevic $S$. Chemical composition and antifungal activity of the essential oils of Lippia rehmanii from South Africa. S Afr J Bot 2010; 76: 37-42.

3. Viljoen AM, Subramoney S, van Vuuren SF, Baser KHC, Demirci $B$. The composition, geographical variation and antimicrobial activity of Lippia javanica (Verbenaceae) leaf essential oils. J Ethnopharmacol 2005; 96: 271-277.

4. Viljoen A. Indigenous South African Medicinal Plants Part 1: Lippia javanica (fever tea). S Afr Pharm J 2007; 74 (5): 50 .

5. Ngassapa O, Runyoro DKB, Harvala E, Chinou IB. Composition and antimicrobial activity of essential oils of two populations of Tanzanian Lippia javanica (Burm. F.) Spreng (Verbenaceae). Flavour Frag J 2003; 18: 221224.

6. Chagonda LS, Makanda DC, Chalchat J. Essential Oils of wild and cultivated Lippia javanica (Spreng) and L. oatesii (Rolfe) from Zimbabwe. J. Essent. Oil Res 2000; 12: 1-6.

7. Leitão SG, Damasceno JP, Martini MG, Miranda SN, Neufeld PM. Analysis of the Chemical Composition and Antimicrobial Activity of the Essential Oil from Lippia triplinervis Gardner (Verbenaceae). J Essent Oil Res 2011: 23: 1-5.

8. Endris A, Asfaw N, Bisrat D. Chemical composition, antimicrobial and antioxidant activities of the essential oil of Lippia javanica leaves from Ethiopia. J Essent Oil Res 2016; 28(3): 221-226.

9. Okoh OO, Sadimenko AP, Afolayan AJ. Comparative evaluation of the antibacterial activities of the essential oils of Rosmarinus officinalis $L$. obtained by hydrodistillation and solvent free microwave extraction methods. Food Chem 2010; 120: 308-312.

10. Kayode RMO, Afolayan AJ. Cytotoxicity and effect of extraction methods on the chemical composition of essential oils of Moringa oleifera seeds. J. Zhejiang Univ-Sci B (Biomed and Biotechnol) 2015; 16(8): 680689.

11. Okoh OO, Afolayan AJ. The effects of hydrodistillation and solvent free microwave extraction methods on the chemical composition and toxicity of essential oils from the leaves of Mentha longiflora L. subsp. capensis. Afr J Pharm Pharmacol 2011; 5(22): 2474-2478.

12. Lucchesi ME, Chemat F, Smadja J. An original solvent free microwave extraction of essential oils from spices. Flavour Frag J 2004a; 19(2): 134-138.

13. Lucchesi ME, Chemat F, Smadja J. Solvent-free microwave extraction of essential oil from aromatic herbs: comparison with conventional hydrodistillation. $J$ Chromatogr A 2004b; 1043(2): 323-327.

Trop J Pharm Res, January 2018; 17(1): 114 
14. Bayramoglu B, Sahin S, Sumnu G. Solvent-free microwave extraction of essential oil from oregano. $J$ Food Eng 2008; 88: 535-540.

15. Adeogun O, Adekunle A, Ashafa A. Chemical composition, lethality and antifungal activities of the extracts of leaf of Thaumatococcus daniellii against foodborne fungi. Beni-Seuf Univ J Appl Sci 2016; 5: 356-368.

16. Alakali JS, Kucha CT, Rabiu IA. Effect of drying temperature on the nutritional quality of Moringa oleifera leaves. Afr J Sci 2015; 9(7): 395-399.

17. Silva SM, Abe SM, Murakami FS, Frensch G, Marquis FA, Nakashima $T$ (2011). Essential oils from different plant parts of Eucalyptus cinera F. Muell. Ex Benth (Myrtaceae) as a source of 1, 8-Cineole and their bioactivities. Pharm 4: 1535-1550.

18. Asekun OT, Grierson DS, Afolayan AJ. Effect of drying methods on the quality and quantity of the essential oil of Mentha longiflora L. subsp. Capensis. Food Chem 2007; 101: 995-998.

19. Al Nomaani RS, Hossain MA, Weli AM, Al-Riyami Q, AlSabahi JN. Chemical composition of essential oils and in vitro antioxidant activity of fresh and dry leaves crude extracts of medicinal plants of Lactuca sativa $L$. native to Sultanate of Oman. Asian Pac J Trop Biomed 2013; 3(5): 353-357.
20. Rahimmalek $M$, Goli SA. Evaluation of six drying treatments with respect to essential oil yield, composition and colour characteristics of Thymys daenensis subsp. daenensis: Calek leaves. Ind Crops Prod; 42: 613-619.

21. Bartolome AP, Villueenor IM, Yang W. Bidens pilosa $L$. (Asteraceae): Botanical properties, traditional uses, phytochemistry and pharmacology. Evid Based Complem Alternat Med 2013; ID 340215

22. Anderson $P$. The effect of ethyl alcohol on the hatching success of Artemia salina. J IEST 2009; 52(1): 9-19.

23. Carballo JL, Hernandez-Inda ZL, Perez P, GarciaGravalos MD. A comparison between two brine shrimp assays to detect in-vitro cytotoxicity in marine natural products. BMC Biotechnol 2002; 2: 17-22.

24. Hamidi MR, Jovanova B, Panovska TK. Toxicological evaluation of the plant products using Brine Shrimp (Artemia salina L.) model. Maced Pharm Bull 2014; 60 (1): 9-18.

25. Clarkson C, Maharaj VJ, Crouch NR, Grace OM, Pillay $P$, Matsabisa MG, Bhagwandin N, Smith PJ, Fobl PI. In vitro antiplasmodial activity of medicinal plants native to or naturalized in South Africa. J Ethnopharm 2004; 92 : 177-191. 